OPEN ACCESS

Edited by:

Egidio lorio,

Istituto Superiore di Sanità, Italy

Reviewed by:

Tariq Shah,

Johns Hopkins University, USA

Samata Kakkad,

Johns Hopkins School of Medicine

*Correspondence:

Francesco Sardanelli

francesco.sardanelli@unimi.it

Specialty section:

This article was submitted

to Cancer Imaging and Diagnosis,

a section of the journal

Frontiers in Oncology

Received: 19 May 2016 Accepted: 30 September 2016

Published: 28 October 2016

Citation:

Sardanelli F, Carbonaro LA, Montemezzi S, Cavedon C and Trimboli RM (2016) Clinical Breast MR Using MRS

or DWI: Who Is the Winner?

Front. Oncol. 6:217.

doi: 10.3389/fonc.2016.00217

\section{Clinical Breast MR Using MRS or DWI: Who Is the Winner?}

\author{
Francesco Sardanelli1,2*, Luca Alessandro Carbonaro', Stefania Montemezzi ${ }^{3}$, \\ Carlo Cavedon ${ }^{4}$ and Rubina Manuela Trimboli ${ }^{1}$
}

\begin{abstract}
${ }^{1}$ Utà di Radiologia, IRCCS Policlinico San Donato, San Donato Milanese, Milan, Italy, ${ }^{2}$ Dipartimento di Scienze Biomediche per la Salute, Università degli Studi di Milano, Milan, Italy, ${ }^{3}$ Dipartimento di Radiologia, Azienda Ospedaliera Universitaria Integrata, Verona, Italy, ${ }^{4}$ Dipartimento di Fisica Sanitaria, Azienda Ospedaliera Universitaria Integrata, Verona, Italy
\end{abstract}

Magnetic resonance imaging (MRI) of the breast gained a role in clinical practice thanks to the optimal sensitivity of contrast-enhanced (CE) protocols. This approach, first proposed 30 years ago and further developed as bilateral highly spatially resolved dynamic study, is currently considered superior for cancer detection to any other technique. However, other directions than CE imaging have been explored. Apart from morphologic features on unenhanced T2-weighted images, two different non-contrast molecular approaches were mainly run in vivo: proton MR spectroscopy (1H-MRS) and diffusion-weighted imaging (DWI). Both approaches have shown aspects of breast cancer (BC) hidden to CE-MRI: $1 \mathrm{H}-\mathrm{MRS}$ allowed for evaluating the total choline peak (tCho) as a biomarker of malignancy; DWI showed that restricted diffusivity is correlated with high cellularity and tumor aggressiveness. Secondary evidence on the two approaches is now available from systematic reviews and meta-analyses, mainly considered in this article: pooled sensitivity ranged $71-74 \%$ for $1 \mathrm{H}-\mathrm{MRS}$ and $84-91 \%$ for DWI; specificity $78-88 \%$ and 75-84\%, respectively. Interesting research perspectives are opened for both techniques, including multivoxel MRS and statistical strategies for classification of MR spectra as well as diffusion tensor imaging and intravoxel incoherent motion for DWI. However, when looking at a clinical perspective, while MRS remained a research tool with important limitations, such as relatively long acquisition times, frequent low quality spectra, difficult standardization, and quantification of tCho tissue concentration, DWI has been integrated in the standard clinical protocols of breast MRI and several studies showed its potential value as a stand-alone approach for $\mathrm{BC}$ detection.

Keywords: breast cancer, diffusion-weighted imaging, magnetic resonance imaging, proton MR spectroscopy, systematic reviews and meta-analyses

\section{INTRODUCTION}

Valuable results of breast magnetic resonance imaging (MRI) were first obtained 30 years ago, when sequences acquired after intravenous injection of a Gd-chelate as a contrast material were added to a non-contrast protocol (1). This approach was further developed as bilateral highly spatially resolved contrast-enhanced (CE) dynamic study, which is now currently considered superior for breast cancer $(\mathrm{BC})$ detection to any other imaging technique with a reported pooled sensitivity and specificity of 93.2 and $71.1 \%$, respectively (2), as also shown by studies on MRI screening of women at increased risk of BC (3-6). In fact, CE-MRI provides a physiopathological information that correlates 
with increased vascularity, vascular permeability, and interstitial space in malignant tissue, not available from mammography and ultrasound. This diagnostic modality has been shown to provide even better sensitivity for BC detection than new techniques such as digital breast tomosynthesis (7-9). As a consequence, CE-MRI has been recommended for a spectrum of indications by medical bodies such as the American College of Radiology (10), the European Society of Breast Imaging $(11,12)$, and the European Society of Breast Cancer Specialists (EUSOMA) (13). The debate is still open on preoperative MRI $(14,15)$, but new indications, such as nipple discharge $(16,17)$ and evaluation of lesions with uncertain malignant potential (so-called B3 lesions), are emerging (18).

However, MR directions other than dynamic CE imaging have been explored searching for a better specificity and a deeper insight into BC biology and metabolism, opening a wider and wider window for the so-called non-contrast breast $M R$. Of note, the clinical relevance of a non-contrast approach for breast MR has been recently fueled by the evidence of Gd accumulation in the brain after multiple administrations of contrast material $(19,20)$, which implies a word of caution for the use of Gd-based contrast at least in the case of screening of healthy subjects when the risk profile is not high.

Traditionally, unenhanced sequences were associated to $\mathrm{CE}$ sequences in a breast MRI protocol for the sake of appraising morphologic features for lesion characterization. Several signs, typically appreciated on T2-weighted images, can be associated with malignancy, the most important being peritumoral and prepectoral edema (21), hook sign, i.e., a hook-like spiculated dendrite from the lesion to the pectoral muscle (22), and necrosis sign, i.e., a hyperintense center in a hypointense lesion (23). Nonenhancing septations are instead usually associated with benign lesions such as fibroadenomas (24).

Two other non-contrast MR methods were mainly implemented for an in vivo study of breast tissues: proton MR spectroscopy (1H-MRS) and diffusion-weighted imaging (DWI).

Proton MR spectroscopy is a non-invasive technique assessing biochemical tissue properties. The presence of a resonance at 3.14-3.34 ppm, attributed to choline metabolites, such as free choline, phosphocholine, and glycerophosphocholine, is usually reported as total choline (tCho). An increased tCho has been detected in malignant breast lesions (25-31), as a result of complex metabolic alteration of biosynthetic and/or catabolic phosphatidylcholine-cycle pathways: the de novo biosynthesis of phosphatidylcholine via the Kennedy pathway and three different major catabolic pathways, contributing to phosphocholine and/ or tCho accumulation (31).

The other non-contrast approach - DWI - is a way for characterizing tissue properties on the basis of the difference in the movement freedom of water molecules (diffusion) along multiple spatial directions, quantified via the measurement of the mean diffusivity and the apparent diffusion coefficient (ADC) (32). Breast malignant tissues show restricted diffusion and significantly lower ADC values compared with those of normal and benign breast tissues (33).

Many studies were published regarding clinical breast applications of MRS and DWI showing a potential for both techniques. The aim of this review is to look at secondary evidence, published between 2010 and 2015, on 1H-MRS and DWI of the breast as well as to try to define which of the two techniques is clinically emerging as a routine tool added to CE-MRI or also potentially usable as a stand-alone approach for cancer detection.

\section{DIAGNOSTIC PERFORMANCE OF 1H-MRS}

The diagnostic performance of $1 \mathrm{H}-\mathrm{MRS}$ of the breast, performed using the single-voxel technique, was evaluated by four systematic reviews (34-37) (Table 1).

The pooled sensitivity ranged between 71 and $74 \%$, and the pooled specificity between 78 and $88 \%$. Baltzer and Dietzel also performed a subgroup analysis for mass and non-mass lesions on six studies obtaining a pooled sensitivity of 68 and $62 \%$, and a pooled specificity of 88 and 69\%, respectively (34), showing that especially for non-mass lesions, $1 \mathrm{H}$-MRS has a too low diagnostic performance to be used in clinical practice. Another subgroup analysis was performed on seven studies by Wang et al. (37), showing that the area under the curve (AUC) at receiver operating characteristic (ROC) analysis was higher (92 vs. 89\%) when tCho signal-to-noise ratio $\geq 2$ was selected as a cutoff for malignancy.

TABLE 1 | Diagnostic performance of proton MR spectroscopy and DWI of the breast as evaluated in systematic reviews and meta-analyses published between 2010 and 2015.

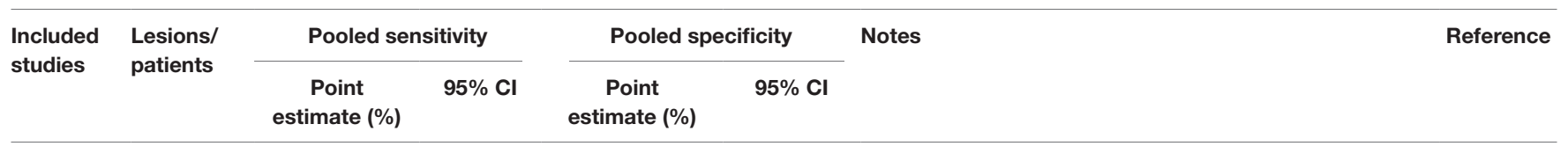

\begin{tabular}{|c|c|c|c|c|c|c|c|}
\hline \multicolumn{8}{|c|}{ Proton MR spectroscopy (1H-MRS) } \\
\hline 19 & $1198 / 1183$ & 73 & $64-82$ & 88 & $85-91$ & tCho evaluation: visual, SNR, 2 or 4 cutoff values & (34) \\
\hline 18 & $1169 / \mathrm{NR}$ & 71 & $68-74$ & 85 & $81-88$ & tCho evaluation: visual, SNR, variable cutoff values & $(35)$ \\
\hline 16 & $1049 / N R$ & 74 & $70-77$ & 78 & $73-82$ & tCho evaluation: visual, SNR, variable cutoff values & $(36)$ \\
\hline 10 & 792/NR & 74 & $69-77$ & 76 & $71-81$ & tCho evaluation: SNR, 2 cut off & $(37)$ \\
\hline \multicolumn{8}{|c|}{ Diffusion-weighted imaging (DWI) } \\
\hline 13 & $964 / 815$ & 84 & $82-87$ & 79 & $75-82$ & Heterogeneity among individual studies; subgroup analysis & $(50)$ \\
\hline 14 & $1276 / 1140$ & 86 & 80-91 & 76 & $67-83$ & Heterogeneity among individual studies; subgroup analysis & (2) \\
\hline 26 & $2151 / 2111$ & 91 & 84-95 & 75 & $61-85$ & Including 11 studies using $b$ values $\leq 600 \mathrm{~mm}^{2} / \mathrm{s}$ & $(51)$ \\
\hline 26 & $2151 / 2111$ & 89 & $85-92$ & 84 & $78-89$ & Including 30 studies using $b$ values $>600 \mathrm{~mm}^{2} / \mathrm{s}$ & $(51)$ \\
\hline
\end{tabular}


All studies included in this meta-analysis (37) showed a high heterogeneity for both sensitivity and specificity, probably due to the large variety of MRS techniques and different field strengths used. Even though improvements from newer technology (such as 3-T magnets and multiple radiofrequency sources) were expected, the authors (37) concluded that none of these innovations significantly influenced the diagnostic performance, as year of publication showed no effect on the diagnostic performance.

Notably, small masses (between 5 and $10 \mathrm{~mm}$ in diameter) or foci $(<5 \mathrm{~mm}$ in diameter) are commonly encountered on CE-MRI, depending on the clinical setting, a proportion of them turning out to be false positives. As a consequence, to avoid work-up (targeted ultrasound, needle biopsy) would be a great achievement. However, in this regard, $1 \mathrm{H}-\mathrm{MRS}$ is scarcely useful. The large majority of studies did not include lesions smaller than $10 \mathrm{~mm}$, thus limiting the applicability of $1 \mathrm{H}-\mathrm{MRS}$ for characterization of small lesions and early BC diagnosis.

One relevant technical problem for $1 \mathrm{H}-\mathrm{MRS}$, especially important for small lesions, is the need for patient's immobility during the acquisition, i.e., for 5-10 min or more (depending also on the time for the preliminary optimization checks), which also impacts on patient's throughput and cost-effectiveness. Of note, a trend for a higher sensitivity of $1 \mathrm{H}-\mathrm{MRS}$ was observed when acquisition is performed before contrast injection, due to a detrimental effect of ionic-charged contrast materials in both experimental and clinical settings (38). As lesion localization (the placement of the spectroscopic volume of interest) has to be made on CE images (especially for otherwise not visible small lesions), the majority of $1 \mathrm{H}-\mathrm{MRS}$ studies were performed after contrast injection, potentially implying a suboptimal sensitivity. On the other hand, a major clinical application of $1 \mathrm{H}-\mathrm{MRS}$ should be lesion characterization, typically arising immediately after the detection, implying that $1 \mathrm{H}-\mathrm{MRS}$ should be performed immediately after CE-MRI, which usually lasts about 15-20 min. Thus, a time prolongation of additional $10 \mathrm{~min}$ increases the probability of patient's movements, reducing the possibility of getting good quality spectra.

Moreover, again especially important for small lesions, to adapt the volume of interest to the lesion morphology is often not easy: cubic or anyway squared volumes of interest tend to include tissues (fat or gland) surrounding the lesion, determining volume contamination and resulting in reduced sensitivity (28). Lesion located posteriorly, close to the pectoral muscle or the thoracic wall, or superficially, near the skin, may provide spectra with low signal-to-noise ratio. Thus, even using 3-T magnets and multiple radiofrequency sources, in clinical practice about $20 \%$ of lesions finally result to be not evaluable with MRS (39).

Several of these limitations are not acting when $1 \mathrm{H}-\mathrm{MRS}$ is applied for the evaluation of the response to neoadjuvant therapy (NAT), typically administered to patients with locally advanced BCs. Studies have shown that $1 \mathrm{H}-\mathrm{MRS}$ enables an early prediction of the final NAT effect (40-42). This is, in our opinion, the most promising clinical application of $1 \mathrm{H}-\mathrm{MRS}$, even though it regards a relatively small fraction of patients. However, also in this field, as we will explain below, DWI is a strong competitor (Figure 1). Anyway, for a well-accepted application of MRS techniques to the NAT setting, large multicenter studies using clearly standardized pathologic response classification are warranted (43-46).

Finally, we should note that $1 \mathrm{H}$-MRS was mostly applied in clinical breast studies using methods not allowing for quantification of tissue tCho concentration. Both the SNR between tCho peak and noise (usually with a $\geq 2$ threshold for malignancy) (34-37) and the integral under the tCho peak (30) have limitations and low potential for standardization. On the other hand, tissue tCho quantification using internal or external standard of reference (47) implies dedicated high technical expertise. This highlights the need for cooperation with a physicist experienced in MRS, not only for tCho tissue quantification but also generally if MRS has to be integrated in a clinical setting. This is a possibility that only large hospitals or academic facilities can afford and is another limitation for MRS effectiveness.

Highly interesting results were obtained, even at $1.5 \mathrm{~T}$, by Stanwell et al. (48), carefully referencing the spectra using a special post-processing: $80 \%$ sensitivity and $100 \%$ specificity. They optimized spectral resolution from human breast tissues resolving the composite choline resonances of the tCho peak. False positives including three lactating women were distinguished by a resonance at 3.28 in contrast with the profile from $\mathrm{BC}$ patients consistent with phosphocholine centered at $3.22 \mathrm{ppm}$. However, a false negative rate of $20 \%$ remained unresolved, and this high spectral resolution was never reproduced, not even using 3-T magnets.

Looking at the potential prognostic value of $1 \mathrm{H}-\mathrm{MRS}$ of the breast, it is worth to note that tCho levels, evaluated by $1 \mathrm{H}-\mathrm{MRS}$, were significantly higher for invasive ductal carcinoma, for cancers with high nuclear or histologic grade, and ER-negative and triple-negative status (49). This perspective deserves confirmation in future large studies with quantification of tissue tCho concentration.

\section{DIAGNOSTIC PERFORMANCE OF DWI}

Breast MR sequences for DWI have been mainly used to try to characterize breast lesions, adding a new parameter in the algorithm for diagnosis, with the potential for avoiding unnecessary biopsy, especially for MRI-only visible lesions that should be sampled under MR-guidance, a time-demanding and high-cost procedure (11).

Studies were performed in order to understand the potential value of DWI added to CE-MRI. Two meta-analyses, from Chen et al. (50) and from Zhang et al. (2), reported an overall sensitivity of 84 and $86 \%$ and an overall specificity of 79 and $76 \%$, respectively. However, a notable heterogeneity among individual studies was observed, and a subgroup analysis to investigate the source of this heterogeneity was performed. The two most important factors resulted to be the $b$ value of diffusion gradient used and the so-called threshold effect (a detectable difference in sensitivities and specificities due to different thresholds used in included studies).

Different $b$ values significantly affect the ADC of breast lesions and therefore confound quantitative DWI. In a large systematic 


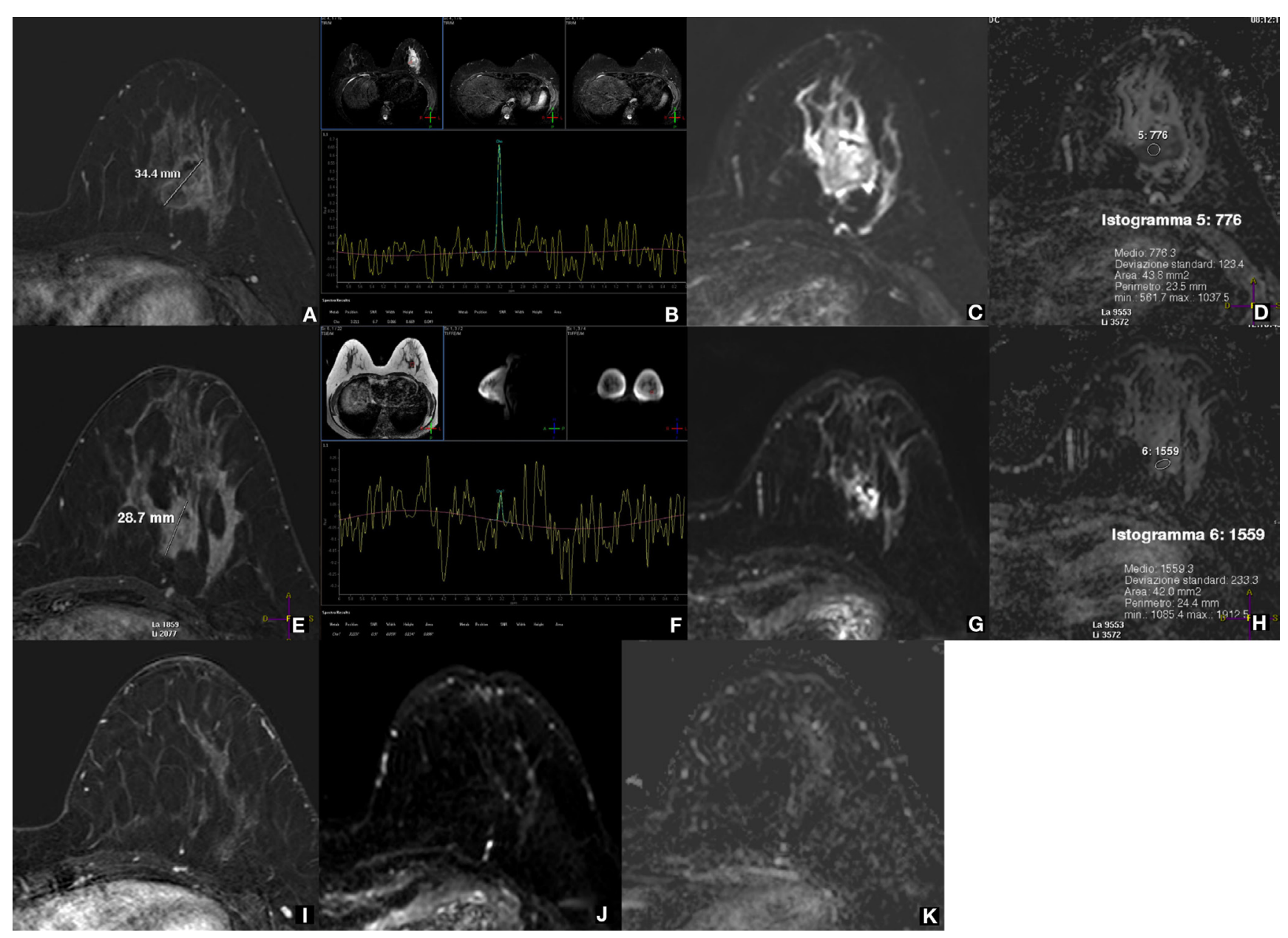

FIGURE 1 | 1H-MRS and DWI for evaluating the response to neoadjuvant therapy (NAT) of a locally advanced breast cancer. A 37 -year-old woman with locally advanced breast cancer before, during, and after neoadjuvant therapy (NAT). Before treatment, the lesion at the lower external quadrant of the left breast is well depicted as a $34.4-\mathrm{mm}$ nodule on subtracted CE-MRI with $0.1 \mathrm{mmol} / \mathrm{kg}$ of gadobenate dimeglumine (A), shows a high tCho peak at $1 \mathrm{H}-\mathrm{MRS}$ with a signal-to-noise ratio (SNR) of 6.7 (B), low diffusivity as a high signal on DWI (C), and a low $\left(0.776 \times 10^{-3} \mathrm{~mm}^{2} / \mathrm{s}\right)$ mean ADC value (D). After two NAT cycles, the lesion is reduced in size $(28.7 \mathrm{~mm})$ at CE-MRI (E), while the tCho peak is no longer detectable at $1 \mathrm{H}-\mathrm{MRS} \mathbf{( F )}$; DWI (G) and ADC map (H) show an evident increased diffusivity (mean ADC $1.559 \times 10^{-3} \mathrm{~mm}^{2} / \mathrm{s}$ ). After the end of treatment, the lesion is not visible at all on CE-MRI (I), DWI (J), and ADC map (K); therefore, MRS was not performed. The lesion was a metaplastic carcinoma with condroid differentiation (negative for estrogen, progesterone, and HER2 receptors, Ki67 $80 \%$ ), and after surgical removal, a complete pathological response was appreciated. Both $1 \mathrm{H}-\mathrm{MRS}$ and DWI early predicted the pathological response to NAT showing an effect more pronounced than that of CE-MRI. Acquisition times: CE-MRI 9 min; 1H-MRS 8 min (including preparation); DWI 5 min (Philips Achieva STx 3.0 T, MultiTransmit radiofrequency technology; dedicated 16-channel breast coil; Azienda Ospedaliera Universitaria Integrata, Verona, Italy).

review performed on 26 studies, including a total of 2111 patients with 2151 breast lesions, Dorrius et al. (51) showed that the combination of $b=0$ and $1000 \mathrm{~s} / \mathrm{mm}^{2}$ is optimal for benign/malignant differentiation. Pooled $89 \%$ sensitivity and $84 \%$ specificity were reported for $b$ values $>600 \mathrm{~s} / \mathrm{mm}^{2}$ (ROC-AUC 0.93), while using $b$ values $\leq 600 \mathrm{~s} / \mathrm{mm}^{2}, 91 \%$ sensitivity and $75 \%$ specificity were obtained (ROC-AUC 0.92). A summary of the recently published meta-analyses on breast DWI is reported in Table $\mathbf{1}$.

There is no clear consensus regarding the threshold values for ADC to diagnose malignancy. A substantial variability of threshold values, ranging $0.90-1.76 \times 10^{-3} \mathrm{~mm}^{2} / \mathrm{s}$ has been observed (51). As a recommendation, a relatively higher threshold value may be used to minimize missing malignancy.
Of note, the studies evaluating mass lesions had a higher specificity (84\%) than those evaluating non-mass lesions (70\%) (2). When added to CE-MRI, DWI sequences showed a significant diagnostic gain: the summary ROC-AUC of CE-MRI combined with DWI was 0.94 compared with 0.85 for CE-MRI alone (2). Differently from $1 \mathrm{H}-\mathrm{MRS}$, contrast injection does not negatively impact on DWI performance (51); ADC measures were reported to not significantly change following DCE-MRI at 3 T, suggesting that DWI and DCE-MRI can be performed in any order without affecting diagnostic performance (52). Moreover, Janka et al. showed that DWI after contrast injection even leads to a slightly better benign/ malignant discrimination (53). 
Furthermore, DWI sequences are not dependent on CE-MRI. They can be performed without previous knowledge of lesion location, as the DWI field of view includes both breasts with a 4- to 5-min acquisition time.

Interestingly, some authors showed as DWI could be used as the main component of an unenhanced (non-contrast) MRI examinations with good sensitivity and high specificity, at least if we consider mass lesions. A reduced detection rate of small mass lesions and of non-mass lesions has to be taken into account (54, 55). However, a sensitivity theoretically comparable with that of screening mammography (54) as well as a potential for avoiding the double reading (55) candidate DWI as a sequence to be used for explorative non-contrast MRI screening studies.

Notably, DWI sequences are undergoing a progressive technical refinement. Spatial resolution and image quality are increasing, allowing also for a more detailed morphology evaluation $(56,57)$. As a matter of fact, authors have positively tried to integrate information coming from DWI into the Breast Imaging Reporting and Data System BI-RADS (58).

Considering the potential prognostic value of DWI, we finally highlight that a recent systematic review demonstrated that breast lesions with increased signal intensity on DWI and decreased signals on ADC are more likely associated to lymph-nodal metastases (59).

\section{RESEARCH TRENDS FOR 1H-MRS AND DWI OF THE BREAST}

First of all, authors attempted to integrate CE-MRI, 1H-MRS, and DWI (multiparametric MRI, mpMRI) to yield significantly higher ROC-AUC (0.936) in comparison with just two of them (0.808) (60). Positron emission tomography (PET) was also added to provide an intriguing mpMRI/PET approach for lesion characterization (61), but we cannot propose this high-cost approach for characterization of breast lesions, when most cases can be solved by a needle sampling.

Regarding $1 \mathrm{H}-\mathrm{MRS}$, two interesting topics are multivoxel spectroscopy and statistical strategies for classifying MR spectra. Multivoxel $1 \mathrm{H}-\mathrm{MRS}$ should overcome the need of a priori knowledge of lesion location (62), allowing for BC screening (or for stratifying $\mathrm{BC}$ risk) on the basis of tCho levels in the healthy gland tissue. However, multivoxel $1 \mathrm{H}-\mathrm{MRS}$ is more technically challenging than single-voxel $1 \mathrm{H}$-MRS for shimming and fat suppression (63). Statistical strategies for classification of spectra were proposed in a $2 \mathrm{D}$ correlated spectroscopy (COSY), where the composite resonances are separated by the use of a second frequency (63). However, this interesting approach seems to be not easy to be reproduced for clinical practice.

Finally, also 31P-MRS (64) was used to provide a direct method for the in vivo detection and quantification of endogenous biomarkers, yielding a new intriguing method for the non-invasive assessment of prognostic and predictive biomarkers in BC treatment.

Regarding DWI, several studies have shown that important information is lost when one relies just on the average ADC and that a higher sensitivity is given by minimum ADC instead of any metrics of the central tendency of ADC values distribution. Mori et al. (65) indicated that a minimum $A D C$ value $<1.1 \times 10^{-3} \mathrm{~mm}^{2} / \mathrm{s}$ and that an $\mathrm{ADC}$ difference (maximum minus minimum $\mathrm{ADC}$ ) greater than $0.23 \times 10^{-3} \mathrm{~mm}^{2} / \mathrm{s}$ suggests the presence of invasive carcinoma in cases with only DCIS at biopsy, a crucial information for patient management.

Second, studies explored the possibility of characterizing axillary lymph nodes using DWI in BC patients. One study (66) considered only nodes with metastases $\geq 5 \mathrm{~mm}$, obtaining 95\% sensitivity and 92\% specificity. More recent studies (67-69) obtained sensitivities from 72 to $85 \%$ and specificities from 80 to $88 \%$. Future multidisciplinary researches should be placed in the context of the current rethinking of axillary dissection (70).

However, the most intriguing research areas for breast DWI are diffusion tensor imaging (DTI) and intravoxel incoherent motion (IVIM). For DTI (which adds information about tissue microstructure by addressing diffusion spatial direction), at least six DWI gradient directions should be applied, so that a symmetrical matrix, the diffusion tensor indeed, can be calculated, describing the anisotropic water diffusion in the tissue. Fractional anisotropy (FA) describes diffusion anisotropy quantitatively on a range from 1 (maximum anisotropy) to 0 (isotropy) (71). Experiences on breast DTI showed that FA does not have incremental value for differential diagnosis over ADC values (71). Eyal et al. (72) developed a breast DTI protocol at $3 \mathrm{~T}$ and image processing means for obtaining vector and parametric maps of the water diffusion tensor. Evaluation of the various diffusion parametric maps indicated that the prime diffusion coefficient and the maximal anisotropy are the most efficient parameters for detecting and diagnosing BC. So far, DTI turned out to be a great tool for visualizing the breast parenchyma but its clinical application remains to be investigated.

The IVIM quantifies molecular diffusion more accurately than $\mathrm{ADC}$ and provides additional information on microperfusion tissue properties. It separates the contribution of T2 from diffusivity using multiple $b$ values, hence being less dependent on the choice of individual $b$ values (73). However, IVIM as well as DTI imply longer acquisition times, and these techniques are not currently suitable for a large use in clinical practice.

\section{CONCLUSION}

The results coming from the secondary evidence about $1 \mathrm{H}-\mathrm{MRS}$ and DWI of the breast are clearly in favor of an easier and more effective use of DWI. If one of the two approaches for non-contrast breast MR has to be chosen for clinical practice, DWI is certainly the winner.

When looking at a clinical perspective, while $1 \mathrm{H}-\mathrm{MRS}$ remained a tool with relevant limitations such as relatively long acquisition times, frequent low quality spectra, difficult standardization and quantification of tissue tCho concentration, DWI is feasible in almost all cases and adds diagnostic power to CE-MRI. Moreover, seminal studies showed the potential value of DWI as a stand-alone approach for BC detection.

As matter of fact, in the context of an ongoing international multicenter study exploring the value of preoperative CE-MRI with over 4500 patients enrolled so far (15), 84\% of them were studied using a technical protocol including DWI among the standard sequences. 


\section{ETHICS STATEMENT}

Did the study presented in the manuscript involve human or animal subjects: Yes. The case presented in Figure $\mathbf{1}$ comes from a prospective clinical study approved by the Ethics Committee of the Azienda Ospedaliera Universitaria Integrata, Verona, Italy. The patient gave written informed consent.

\section{REFERENCES}

1. Heywang SH, Hahn D, Schmidt H, Krischke I, Eiermann W, Bassermann R, et al. MR imaging of the breast using gadolinium-DTPA. J Comput Assist Tomogr (1990) 10:199-204. doi:10.1097/00004728-198603000-00005

2. Zhang L, Tang M, Min Z, Lu J, Lei X, Zhang X. Accuracy of combined dynamic contrast-enhanced magnetic resonance imaging and diffusion-weighted imaging for breast cancer detection: a meta-analysis. Acta Radiol (2016) 57:651-60. doi:10.1177/0284185115597265

3. Kuhl C, Weigel S, Schrading S, Arand B, Bieling H, König R, et al. Prospective multicenter cohort study to refine management recommendations for women at elevated familial risk of breast cancer: the EVA trial. J Clin Oncol (2010) 28:1450-7. doi:10.1200/JCO.2009.23.0839

4. Sardanelli F, Podo F, Santoro F, Manoukian S, Bergonzi S, Trecate G, et al. Multicenter surveillance of women at high genetic breast cancer risk using mammography, ultrasonography, and contrast-enhanced magnetic resonance imaging (the high breast cancer risk Italian 1 study): final results. Invest Radiol (2011) 46:94-105. doi:10.1097/RLI.0b013e3181f3fcdf

5. Santoro F, Podo F, Sardanelli F. MRI screening of women with hereditary predisposition to breast cancer: diagnostic performance and survival analysis. Breast Cancer Res Treat (2014) 147:685-7. doi:10.1007/s10549-014-3097-1

6. Podo F, Santoro F, Di Leo G, Manoukian S, de Giacomi C, Corcione S, et al. Triple-negative versus non-triple-negative breast cancers in high-risk women: phenotype features and survival from the HIBCRIT-1 MRI-including screening study. Clin Cancer Res (2016) 22:895-904. doi:10.1158/1078-0432. CCR-15-0459

7. Mariscotti G, Houssami N, Durando M, Bergamasco L, Campanino PP, Ruggieri C, et al. Accuracy of mammography, digital breast tomosynthesis, ultrasound and MR imaging in preoperative assessment of breast cancer. Anticancer Res (2014) 34:1219-25.

8. Kim WH, Chang JM, Moon H-G, Yi A, Koo HR, Gweon HM, et al. Comparison of the diagnostic performance of digital breast tomosynthesis and magnetic resonance imaging added to digital mammography in women with known breast cancers. Eur Radiol (2016) 26:1556-64. doi:10.1007/s00330-015-3998-3

9. Clauser P, Carbonaro LA, Pancot M, Girometti R, Bazzocchi M, Zuiani C, et al. Additional findings at preoperative breast MRI: the value of second-look digital breast tomosynthesis. Eur Radiol (2015) 25:2830-9. doi:10.1007/ s00330-015-3720-5

10. American College of Radiology. ACR Practice Parameter for the Performance of Contrast-Enhanced Magnetic Resonance Imaging (MRI) of the Breast (2013). Available from: http://www.acr.org/ /media/2a0eb28eb59041e2825179afb72ef624.pdf

11. Mann RM, Kuhl CK, Kinkel K, Boetes C. Breast MRI: guidelines from the European Society of Breast Imaging. Eur Radiol (2008) 18:1307-18. doi:10.1007/s00330-008-0863-7

12. Mann RM, Balleyguier C, Baltzer PA, Bick U, Colin C, Cornford E, et al. Breast MRI: EUSOBI recommendations for women's information. Eur Radiol (2015) 25:3669-78. doi:10.1007/s00330-015-3807-z

13. Sardanelli F, Boetes C, Borisch B, Decker T, Federico M, Gilbert FJ, et al. Magnetic resonance imaging of the breast: recommendations from the EUSOMA working group. Eur J Cancer (2010) 46:1296-316. doi:10.1016/ j.ejca.2010.02.015

14. Sardanelli F, Trimboli RM. Preoperative MRI: did randomized trials conclude the debate? Eur J Radiol (2012) 81(Suppl 1):S135-6. doi:10.1016/ S0720-048X(12)70056-6

15. Trimboli RM, Di Leo G, Sacchetto D, Mann R, Álvarez Benito M, Zuiani C, et al. The impact of breast MRI on surgical planning and reoperation rate:

\section{AUTHOR CONTRIBUTIONS}

FS is responsible for the conception of the work. LC and RT searched for literature focusing on secondary evidence. FS, LC, and RT drafted multiple versions of the manuscript. SM and CC revised the manuscript adding content of intellectual importance. All authors approved the final version of the manuscript.

first results from the MIPA study. Insights Imag (2016) 7(Suppl 1):S464-5; Proceedings of the European Congress of Radiology; 2016 Mar 2-6; Vienna, Austria. doi:10.1007/s13244-016-0475-8

16. Bahl M, Baker JA, Greenup RA, Ghate SV. Evaluation of pathologic nipple discharge: what is the added diagnostic value of MRI? Ann Surg Oncol (2015) 22(Suppl 3):S435-41. doi:10.1245/s10434-015-4792-9

17. Sanders LM, Daigle M. The rightful role of MRI after negative conventional imaging in the management of bloody nipple discharge. Breast J (2016) 22:209-12. doi:10.1111/tbj.12551

18. Londero V, Zuiani C, Linda A, Girometti R, Bazzocchi M, Sardanelli F. Highrisk breast lesions at imaging-guided needle biopsy: usefulness of MRI for treatment decision. AJR Am J Roentgenol (2012) 199:W240-50. doi:10.2214/ AJR.11.7869

19. Kanda T, Ishii K, Kawaguchi H, Kitajima K, Takenaka D. High signal intensity in the dentate nucleus and globus pallidus on unenhanced T1-weighted MR images: relationship with increasing cumulative dose of a gadolinium-based contrast material. Radiology (2014) 270:834-41. doi:10.1148/radiol.13131669

20. Kanda T, Fukusato T, Matsuda M, Toyoda K, Oba H, Kotoku J, et al. Gadolinium-based contrast agent accumulates in the brain even in subjects without severe renal dysfunction: evaluation of autopsy brain specimens with inductively coupled plasma mass spectroscopy. Radiology (2015) 276:228-32. doi:10.1148/radiol.2015142690

21. Uematsu T. Focal breast edema associated with malignancy on T2-weighted images of breast MRI: peritumoral edema, prepectoral edema, and subcutaneous edema. Breast Cancer (2015) 22:66-70. doi:10.1007/s12282-014-0572-9

22. Dietzel M, Baltzer PAT, Vag T, Gajda M, Camara O, Kaiser WA. The hook sign for differential diagnosis of malignant from benign lesions in magnetic resonance mammography: experience in a study of 1084 histologically verified cases. Acta Radiol (2010) 51:137-43. doi:10.3109/02841850903463638

23. Dietzel M, Baltzer PAT, Vag T, Herzog A, Gajda M, Camara O, et al. The necrosis sign in magnetic resonance-mammography: diagnostic accuracy in 1,084 histologically verified breast lesions. Breast J (2010) 16:603-8. doi:10.1111/j.1524-4741.2010.00982.x

24. Malich A, Fischer DR, Wurdinger S, Boettcher J, Marx C, Facius M, et al. Potential MRI interpretation model: differentiation of benign from malignant breast masses. Am J Roentgenol (2005) 185:964-70. doi:10.2214/AJR.04.1073

25. Fausto A, Magaldi A, Babaei Paskeh B, Menicagli L, Lupo EN, Sardanelli F. MR imaging and proton spectroscopy of the breast: how to select the images useful to convey the diagnostic message. Radiol Med (2007) 112:1060-8. doi:10.1007/ s11547-007-0193-x

26. Bartella L, Morris EA, Dershaw DD, Liberman L, Thakur SB, Moskowitz C, et al. Proton MR spectroscopy with choline peak as malignancy marker improves positive predictive value for breast cancer diagnosis: preliminary study. Radiology (2006) 239:686-92. doi:10.1148/radiol.2393051046

27. Katz-Brull R, Lavin PT, Lenkinski RE. Clinical utility of proton magnetic resonance spectroscopy in characterizing breast lesions. J Natl Cancer Inst (2002) 94:1197-203. doi:10.1093/jnci/94.16.1197

28. Sardanelli F, Fausto A, Podo F. MR spectroscopy of the breast. Radiol Med (2008) 113:56-64. doi:10.1007/s11547-008-0228-y

29. Sardanelli F, Fausto A, Di Leo G, De Nijs R, Vorbuchner M, Podo F. In vivo proton MR spectroscopy of the breast using the total choline peak integral as a marker of malignancy. Am J Roentgenol (2009) 192:1608-17. doi:10.2214/ AJR.07.3521

30. Bartella L, Huang W. Proton ( $1 \mathrm{H})$ MR spectroscopy of the breast. Radiographics (2007) 27(Suppl 1):S241-52. doi:10.1148/rg.27si075504

31. Podo F, Sardanelli F, Iorio E, Canese R, Carpinelli G, Fausto A, et al. Abnormal choline phospholipid metabolism in breast and ovary cancer: molecular bases 
for noninvasive imaging approaches. Curr Med Imag Rev (2007) 3:123-37. doi:10.2174/157340507780619160

32. Iima M, Le Bihan D. Clinical intravoxel incoherent motion and diffusion MR imaging: past, present, and future. Radiology (2016) 278:13-32. doi:10.1148/ radiol.2015150244

33. Woodhams R, Ramadan S, Stanwell P, Sakamoto S, Hata H, Ozaki M, et al. Diffusion-weighted imaging of the breast: principles and clinical applications. Radiographics (2011) 31:1059-84. doi:10.1148/rg.314105160

34. Baltzer PAT, Dietzel M. Breast lesions: diagnosis by using proton MR spectroscopy at 1.5 and $3.0 \mathrm{~T}$ - systematic review and meta-analysis. Radiology (2013) 267:735-46. doi:10.1148/radiol.13121856

35. Cen D, Xu L. Differential diagnosis between malignant and benign breast lesions using single-voxel proton MRS: a meta-analysis. J Cancer Res Clin Oncol (2014) 140:993-1001. doi:10.1007/s00432-014-1605-7

36. Tan J, Xu L, Yao W, Wan Y, Zhou S, Xin SX. In vivo post-contrast 1H-MRS evaluation of malignant and benign breast lesions: a meta-analysis. Tumour Biol (2015) 36:345-52. doi:10.1007/s13277-014-2635-0

37. Wang X, Wang XJ, Song HS, Chen LH. 1H-MRS evaluation of breast lesions by using total choline signal-to-noise ratio as an indicator of malignancy: a meta-analysis. Med Oncol (2015) 32:160. doi:10.1007/s12032-015-0603-1

38. Lenkinski RE, Wang X, Elian M, Goldberg SN. Interaction of gadolinium-based MR contrast agents with choline: implications for MR spectroscopy (MRS) of the breast. Magn Reson Med (2009) 61:1286-92. doi:10.1002/mrm.21937

39. Montemezzi S, Caumo F, Baglio I, Camera L, Meliado G, Cavedon C. MR spectroscopy of the breast at 3 Tesla: a clinical experience. Proceedings of the Radiological Society of North America 2013 Scientific Assembly and Annual Meeting, December 1-December 6, 2013. Chicago, IL (2013). VSBR21-17. Available from: http://archive.rsna.org/2013/13021676.html

40. Manton DJ, Chaturvedi A, Hubbard A, Lind MJ, Lowry M, Maraveyas A, et al. Neoadjuvant chemotherapy in breast cancer: early response prediction with quantitative MR imaging and spectroscopy. Br J Cancer (2006) 94:427-35. doi:10.1038/sj.bjc.6602948

41. Baek H-M, Chen J-H, Nie K, Yu HJ, Bahri S, Mehta RS, et al. Predicting pathologic response to neoadjuvant chemotherapy in breast cancer by using MR imaging and quantitative 1H MR spectroscopy. Radiology (2009) 251:653-62. doi:10.1148/radiol.2512080553

42. Leong KM, Lau P, Ramadan S. Utilisation of MR spectroscopy and diffusion weighted imaging in predicting and monitoring of breast cancer response to chemotherapy. J Med Imaging Radiat Oncol (2015) 59:268-77. doi:10.1111/1754-9485.12310

43. Marinovich ML, Houssami N, Macaskill P, Sardanelli F, Irwig L, Mamounas EP, et al. Meta-analysis of magnetic resonance imaging in detecting residual breast cancer after neoadjuvant therapy. J Natl Cancer Inst (2013) 105:321-33. doi:10.1093/jnci/djs528

44. Marinovich ML, Macaskill P, Irwig L, Sardanelli F, von Minckwitz G, Mamounas E, et al. Meta-analysis of agreement between MRI and pathologic breast tumour size after neoadjuvant chemotherapy. Br J Cancer (2013) 109:1528-36. doi:10.1038/bjc.2013.473

45. Marinovich ML, Sardanelli F, Ciatto S, Mamounas E, Brennan M, Macaskill P, et al. Early prediction of pathologic response to neoadjuvant therapy in breast cancer: systematic review of the accuracy of MRI. Breast (2012) 21:669-77. doi:10.1016/j.breast.2012.07.006

46. Marinovich ML, Macaskill P, Irwig L, Sardanelli F, Mamounas E, von Minckwitz G, et al. Agreement between MRI and pathologic breast tumor size after neoadjuvant chemotherapy, and comparison with alternative tests: individual patient data meta-analysis. BMC Cancer (2015) 15:662. doi:10.1186/ s12885-015-1664-4

47. Bolan PJ. Magnetic resonance spectroscopy of the breast: current status. Magn Reson Imaging Clin N Am (2013) 21:625-39. doi:10.1016/j.mric.2013.04.008

48. Stanwell P, Gluch L, Clark D, Tomanek B, Baker L, Giuffrè B, et al. Specificity of choline metabolites for in vivo diagnosis of breast cancer using $1 \mathrm{H}$ MRS at 1.5 T. Eur Radiol (2005) 15:1037-43. doi:10.1007/s00330-004-2475-1

49. Shin HJ, Baek H-M, Cha JH, Kim HH. Evaluation of breast cancer using proton MR spectroscopy: total choline peak integral and signal-to-noise ratio as prognostic indicators. AJR Am J Roentgenol (2012) 198:W488-97. doi:10.2214/ AJR.11.7292

50. Chen X, Li W, Zhang Y, Wu Q, Guo Y, Bai Z. Meta-analysis of quantitative diffusion-weighted MR imaging in the differential diagnosis of breast lesions. BMC Cancer (2010) 10:693. doi:10.1186/1471-2407-10-693
51. Dorrius MD, Dijkstra H, Oudkerk M, Sijens PE. Effect of b value and pre-admission of contrast on diagnostic accuracy of 1.5-T breast DWI: a systematic review and meta-analysis. Eur Radiol (2014) 24:2835-47. doi:10.1007/ s00330-014-3338-z

52. Nguyen VT, Rahbar H, Olson ML, Liu C-L, Lehman CD, Partridge SC. Diffusion-weighted imaging: effects of intravascular contrast agents on apparent diffusion coefficient measures of breast malignancies at 3 Tesla. J Magn Reson Imaging (2015) 42:788-800. doi:10.1002/jmri.24844

53. Janka R, Hammon M, Geppert C, Nothhelfer A, Uder M, Wenkel E. Diffusionweighted MR imaging of benign and malignant breast lesions before and after contrast enhancement. RöFo (2014) 186:130-5. doi:10.1055/s-0033-1350298

54. Baltzer PAT, Benndorf M, Dietzel M, Gajda M, Camara O, Kaiser WA. Sensitivity and specificity of unenhanced MR mammography (DWI combined with T2-weighted TSE imaging, ueMRM) for the differentiation of mass lesions. Eur Radiol (2010) 20:1101-10. doi:10.1007/s00330-009-1654-5

55. Trimboli RM, Verardi N, Cartia F, Carbonaro LA, Sardanelli F. Breast cancer detection using double reading of unenhanced MRI including T1-weighted, T2-weighted STIR, and diffusion-weighted imaging: a proof of concept study. AJR Am J Roentgenol (2014) 203:674-81. doi:10.2214/AJR.13.11816

56. Wisner DJ, Rogers N, Deshpande VS, Newitt DN, Laub GA, Porter DA, et al. High-resolution diffusion-weighted imaging for the separation of benign from malignant BI-RADS $4 / 5$ lesions found on breast MRI at 3T. J Magn Reson Imaging (2014) 40:674-81. doi:10.1002/jmri.24416

57. Barentsz MW, Taviani V, Chang JM, Ikeda DM, Miyake KK, Banerjee S, et al. Assessment of tumor morphology on diffusion-weighted (DWI) breast MRI: diagnostic value of reduced field of view DWI. J Magn Reson Imaging (2015) 42:1656-65. doi:10.1002/jmri.24929

58. Pinker K, Bickel H, Helbich TH, Gruber S, Dubsky P, Pluschnig U, et al. Combined contrast-enhanced magnetic resonance and diffusion-weighted imaging reading adapted to the "Breast Imaging Reporting and Data System" for multiparametric 3-T imaging of breast lesions. Eur Radiol (2013) 23:1791-802. doi:10.1007/s00330-013-2771-8

59. Sun J-H, Jiang L, Guo F, Zhang X-S. Diagnostic significance of apparent diffusion coefficient values with diffusion weighted MRI in breast cancer: a meta-analysis. Asian Pac J Cancer Prev (2014) 15:8271-7. doi:10.7314/ APJCP.2014.15.19.8271

60. Pinker K, Bogner W, Baltzer P, Gruber S, Bickel H, Brueck B, et al. Improved diagnostic accuracy with multiparametric magnetic resonance imaging of the breast using dynamic contrast-enhanced magnetic resonance imaging, diffusion-weighted imaging, and 3-dimensional proton magnetic resonance spectroscopic imaging. Invest Radiol (2014) 49:421-30. doi:10.1097/ RLI.0000000000000029

61. Pinker K, Bogner W, Baltzer P, Karanikas G, Magometschnigg H, Brader P, et al. Improved differentiation of benign and malignant breast tumors with multiparametric 18fluorodeoxyglucose positron emission tomography magnetic resonance imaging: a feasibility study. Clin Cancer Res (2014) 20:3540-9. doi:10.1158/1078-0432.CCR-13-2810

62. Sijens PE, Dorrius MD, Kappert P, Baron P, Pijnappel RM, Oudkerk M. Quantitative multivoxel proton chemical shift imaging of the breast. Magn Reson Imaging (2010) 28:314-9. doi:10.1016/j.mri.2009.11.004

63. Birdwell RL, Mountford CE, Iglehart JD. Molecular imaging of the breast. Radiol Clin North Am (2010) 48:1075-88. doi:10.1016/j.rcl.2010.07.017

64. Klomp DWJ, van de Bank BL, Raaijmakers A, Korteweg MA, Possanzini C, Boer VO, et al. 31P MRSI and $1 \mathrm{H}$ MRS at $7 \mathrm{~T}$ : initial results in human breast cancer. NMR Biomed (2011) 24:1337-42. doi:10.1002/nbm.1696

65. Mori N, Ota H, Mugikura S, Takasawa C, Tominaga J, Ishida T, et al. Detection of invasive components in cases of breast ductal carcinoma in situ on biopsy by using apparent diffusion coefficient MR parameters. Eur Radiol (2013) 23:2705-12. doi:10.1007/s00330-013-2902-2

66. Fornasa F, Nesoti MV, Bovo C, Bonavina MG. Diffusion-weighted magnetic resonance imaging in the characterization of axillary lymph nodes in patients with breast cancer. J Magn Reson Imaging (2012) 36:858-64. doi:10.1002/ jmri.23706

67. Luo N, Su D, Jin G, Liu L, Zhu X, Xie D, et al. Apparent diffusion coefficient ratio between axillary lymph node with primary tumor to detect nodal metastasis in breast cancer patients. J Magn Reson Imaging (2013) 38:824-8. doi:10.1002/jmri.24031

68. Rautiainen S, Könönen M, Sironen R, Masarwah A, Sudah M, Hakumäki J, et al. Preoperative axillary staging with 3.0-T breast MRI: clinical value of 
diffusion imaging and apparent diffusion coefficient. PLoS One (2015) 10:e0122516. doi:10.1371/journal.pone.0122516

69. Yamaguchi K, Schacht D, Nakazono T, Irie H, Abe H. Diffusion weighted images of metastatic as compared with nonmetastatic axillary lymph nodes in patients with newly diagnosed breast cancer. J Magn Reson Imaging (2015) 42:771-8. doi:10.1002/jmri.24829

70. Li CZ, Zhang P, Li RW, Wu CT, Zhang XP, Zhu HC. Axillary lymph node dissection versus sentinel lymph node biopsy alone for early breast cancer with sentinel node metastasis: a meta-analysis. Eur J Surg Oncol (2015) 41:958-66. doi:10.1016/j.ejso.2015.05.007

71. Baltzer PAT, Schäfer A, Dietzel M, Grässel D, Gajda M, Camara O, et al. Diffusion tensor magnetic resonance imaging of the breast: a pilot study. Eur Radiol (2011) 21:1-10. doi:10.1007/s00330-010-1901-9

72. Eyal E, Shapiro-Feinberg M, Furman-Haran E, Grobgeld D, Golan T, Itzchak Y, et al. Parametric diffusion tensor imaging of the breast. Invest Radiol (2012) 47:284-91. doi:10.1097/RLI.0b013e3182438e5d
73. Dijkstra H, Dorrius MD, Wielema M, Jaspers K, Pijnappel RM, Oudkerk M, et al. Semi-automated quantitative intravoxel incoherent motion analysis and its implementation in breast diffusion-weighted imaging. J Magn Reson Imaging (2016) 43:1122-31. doi:10.1002/jmri.25086

Conflict of Interest Statement: The authors declare that the research was conducted in the absence of any commercial or financial relationships that could be construed as a potential conflict of interest.

Copyright (C) 2016 Sardanelli, Carbonaro, Montemezzi, Cavedon and Trimboli. This is an open-access article distributed under the terms of the Creative Commons Attribution License (CC BY). The use, distribution or reproduction in other forums is permitted, provided the original author(s) or licensor are credited and that the original publication in this journal is cited, in accordance with accepted academic practice. No use, distribution or reproduction is permitted which does not comply with these terms 